\title{
Ocean Monitoring with SAR: An Overview
}

\author{
Gerardo Di Martino, Antonio Iodice, Daniele Riccio, Giuseppe Ruello \\ DIETI - Università di Napoli Federico II \\ Napoli, Italy
}

\begin{abstract}
In this paper an overview of Synthetic Aperture Radar (SAR) applications for the monitoring of the ocean surface is presented. In particular, the necessity of adequate direct models for the description of the sea surface and of its interaction with the incident electromagnetic field is stressed and suitable models are introduced. Based on the introduced models, two main applications are analyzed in detail, i.e. the monitoring of oil slicks on the sea surface and the mitigation of azimuth ambiguities, which is crucial in support of ship-detection applications.
\end{abstract}

Keywords-Synthetic Aperture Radar (SAR), electromagnetic modeling.

\section{INTRODUCTION}

Modern spaceborne Synthetic Aperture Radar (SAR) systems (e.g. TerraSAR-X, Cosmo/SkyMed and Sentinel-1) are designed to generate synoptic views of the Earth and ocean surfaces with unprecedented wide coverages leading to revisit times as small as one day or some hours [1]. This characteristic is particularly important for maritime applications, which involve the monitoring of dynamic targets and phenomena over extremely large areas. In most cases the requirements are not too strict in terms of resolution, whereas the required revisit times in maritime surveillance applications are very small, both when the focus is on ship detection and on oil-spill monitoring and tracking.

SAR is an active sensor operating at microwave frequencies, thus providing the opportunity to obtain images of the Earth in all-weather, all-time conditions. The possibility to monitor the oceans with SAR is contingent upon the availability of adequate physical electromagnetic models able to account for the interaction of the incident electromagnetic field with the sea surface, under a wide variety of potential surface conditions [2]. For the sake of simplicity and to emphasize the presentation framework, only the main stripmap SAR products are considered in the present paper; furthermore, only single-polarization, single-acquisition (along- and across track- interferometry, are not taken into account), as well as, no focus-adjusted or motion-compensated images are considered [1].

In contrast to natural rough soil surfaces' imaging, which must be described using almost-unpredictable mostlystochastic physical and electromagnetic models [3]-[4], for the ocean surface well-behaved quite-regular partly-deterministic models can be effectively used [5]. In particular, the paper rationale relies on describing interaction between the SAR transmitted electromagnetic wave and the ocean surface spectra. This description is arranged in terms of the different regions of the ocean spectra (from smaller to larger spatial scales) as evident at the different spatial scales dealt with in the SAR image. Once the description of the ocean surface geometry is available adequate evaluation of the electromagnetic scattering must be performed. For the case of interest the main mechanism involved is the Bragg phenomenon, which accounts for the resonant behavior due to the presence of capillary or gravity-capillary ocean waves, i.e. those waves presenting a wavelength similar to the electromagnetic one. Finally, appropriate solutions must be enforced to account for the presence of the speckle phenomenon. This can be accomplished introducing a correlation between neighboring pixels [5] and through the use of adequate stochastic models [6]. These topics, which provide overall direct models for ocean surface imaging in standard conditions, are discussed in Section II.

The above mentioned framework provides a complete quantitative analysis of SAR ocean images under standard conditions. But, quite remarkably, it allows conveniently discussing the intriguing cases of ocean monitoring under special circumstances. In the present paper two specific (not standard) cases are considered in Section III. First, it is shown how to model, on physical bases via the Marangoni effect [7], the appearance of oil slicks on the ocean surface. This model is developed and presented to quantitatively support mainly the oil slick detection procedure directly via SAR images. Second, the ship-detection problem is considered and, more specifically, a pre-processing technique based on the same SAR system models introduced for SAR data simulation is presented. The presented approach is aimed at the suppression of the azimuth ambiguity effects, directly working in the meaningful Fourier transformed domain.

\section{DIRECT MODELS FOR OCEAN SURFACE IMAGING}

Information extraction from SAR images of the ocean surface can be accomplished only on the basis of complete models relating relevant scene parameters, such as sea wave parameters (which are in turn related to wind speed and direction), to the final SAR raw signal. These direct models can be roughly divided into surface models and electromagnetic models; however, the two models are not independent and a specific surface model calls for adequate electromagnetic models. In this section we present the main surface and electromagnetic models for the description of the imaging of the ocean surface. Moreover, we also discuss some specific details regarding SAR raw signal simulation of ocean scenes. 


\section{A. Ocean Surface Spectrum}

A specific realization of the ocean surface is the result of involved non-linear hydrodynamic phenomena generating surface waves, whose wavelengths range from millimeter to kilometer scale. A first classification of ocean waves can be done according to their spatial length, where the range goes from short waves with length of less than one meter to long waves of several hundred meters length, whereas intermediate waves present lengths in the order of tens of meters [8].

However, ocean waves can also be described according to the physical mechanism dictating their formation [8]. In particular, capillary waves (centimeter wavelengths) are the first to rise in response to local winds; then the capillary waves begin to transfer energy to waves presenting longer wavelengths, giving rise, first, to gravity-capillary waves (one centimeter to decimeters wavelengths) and, then, to gravity waves (which correspond to intermediate and long waves). The reason of their names can be better understood looking at the dispersion relation [7]-[8], which is the starting point for the study of ocean wave propagation:

$$
\omega(k)=\sqrt{g k \tanh \left(k h_{0}\right)+\frac{\tau}{\rho} k^{3}}
$$

where $\omega$ is the angular frequency of the ocean wave; $g$ is the gravity acceleration; $\tau$ is the surface tension; $\rho$ is the water density; $h_{0}$ is the sea depth; and $k$ is the surface wavenumber. The dispersion equation (1) clearly shows that, while long waves (small $k$, which implies that the second term under square root can be ignored) are governed by the gravity force, very short waves (very large $k$ ) are related to the surface tension. Note that the transfer of energy from lower wavelengths waves to larger wavelengths ones continues until equilibrium is reached (fully developed sea).

The ocean surface roughness is usually statistically modeled as a Gaussian stationary process $z(x, y)$ with pdf

$$
p_{z}(\zeta)=\frac{1}{\sqrt{2 \pi} \sigma} e^{-\frac{\zeta^{2}}{2 \sigma^{2}}}
$$

where $\sigma$ is the standard deviation. This provides a first order characterization of the surface.

The second order characterization is accomplished through appropriate spatial and temporal autocorrelation functions. In this case the statistical stationarity assumption allows us to define corresponding spectral quantities. In particular, the twodimensional directional spectrum $S(k, \theta)$ (in polar coordinates) is obtained as the Fourier transform of the spatial autocorrelation function, while the one-dimensional power spectral density $E(\omega)$ is the Fourier transform of the temporal autocorrelation function. The spectrum $E(\omega)$ can be obtained from $S(k, \theta)$ by integration over the $\theta$ coordinate, taking into account the dispersion relation (1)

$$
E(\omega)=\frac{1}{2 \pi} \int_{-\pi}^{\pi} S[k(\omega), \theta] \frac{d k}{d \omega} d \theta
$$

The directional spectrum is usually factorized as the product of a function $S_{k}(k)$ only depending on $k$ and another function which mainly depends on $\theta$, while is only marginally dependent on $k$.

In the specialized literature several models have been proposed for the spectrum $S_{k}(k)$. For the case of SAR we need an accurate description of the ocean surface at wavelengths typical of gravity-capillary and capillary waves, which are the ones that, presenting wavelengths of the same order of the incident microwave electromagnetic waves, mostly interact with the impinging signal. The modified Pearson-Moskowitz spectrum, which is tailored to a fully developed sea, is adequate to the scope [7]. It provides a model of the sea surface spectrum over a wide range of wavelengths. In particular, the capillary waves are described through a power-law fractal spectrum.

\section{B. Electromagnetic Model}

The dominant mechanism dictating the scattering from the ocean surface is the Bragg resonance in presence of oblique incidence. In fact, the Small Perturbation Method analytical solution of the scattering problem can be considered [4], [7]. According to this approach the Normalized Radar Cross Section (NRCS) $\sigma_{0}$ is dependent on the sea surface directional spectrum

$$
\sigma_{p q}^{0}=\frac{4}{\pi} k_{0}^{4} \cos ^{4} \vartheta\left|\alpha_{p q}(\vartheta)\right|^{2} S\left(2 k_{0} \sin \vartheta, 0\right)
$$

where $k_{0}$ is the electromagnetic wavenumber; $\vartheta$ is the local incidence angle; and $\alpha_{p q}$ (where $p$ and $q$ can assume the value $h$ and $v$, standing for vertical and horizontal polarizations, respectively) is a coefficient dependent on the field polarization [7]. Hence, according to the Bragg phenomenon described by (4), the SAR sensor acts as a frequency selector, so that only sea waves at resonant wavelengths contribute to the scattering. The appropriate spectrum can be used according to the discussion reported in the previous sub-section. We note that, whenever the case, the Physical Optics solution within the Kirchhoff approximation regime can be used. Similarly to the SPM, also in this case the NRCS depends on the meaningful sea surface directional spectrum [4].

Some final comments are necessary regarding the speckle effect, which is present in SAR image due to the coherent character of the returns. The speckle can be modeled in a statistical way: the most appropriate model for the ocean case is the $\mathrm{K}$ distribution [9]. The shape of this distribution depends on the effective number of scatterers present in the resolution cell. In [6] we showed that this parameter is related to the physical parameters describing the roughness of the imaged surface. In addition, note that, depending on the considered sensor resolution and sampling frequencies, a certain degree of correlation among neighboring pixels can be present, as evident on actual SAR image. 


\section{SAR Raw Signal Simulation}

The simulation of the SAR raw signal of the ocean surface calls for special attention, due to the time-varying nature of the reflectivity of the ocean. In order to circumvent this problem the Harger Distributed Surface model can be used so that the time dependence can appropriately "migrate" from the reflectivity function to the impulse response function of the system making use of the dispersion relation for the long wave spectrum [5], [7] (see discussion in Section II.A). Once this is accomplished, SAR simulation can be performed via usual FFT algorithms, taking into account appropriate interpolation schemes [5].

\section{OCEAN MONITORING}

In the following two specific oceanic SAR applications are discussed in detail. Use is made of appropriate modifications of the above introduced models, demonstrating their flexibility and their value in the context of SAR maritime applications.

\section{A. Oil Slicks Simulation and Detection}

The presence of oil slicks on the sea surface can be due to both natural and human-induced (potentially illegal) phenomena. In any case, appropriate monitoring techniques are advisable for identification and tracking. In particular, remote sensing instruments (and in particular SAR) provide the necessary coverage and revisit times necessary to perform an effective monitoring activity [10].

The models introduced in Section II are appropriate to describe the ocean surface in standard conditions. Whenever an oil film is present on the surface the models must be modified to take into account the reduction of surface tension and friction between wind and liquid surface. In this situation, the wind can transfer a smaller energy to capillary waves in oilslick-covered areas. In addition, viscous dissipation increases. These phenomena cause a decrement of the capillary spectrum intensity, implying a weaker electromagnetic return from areas where oil is present.

Quantification of the above mentioned effects can be obtained using Marangoni theory [7], [11]. According to Marangoni, surfaces covered by a slick can carry two kinds of waves: the gravity-capillary and the Marangoni waves. Marangoni waves are related to surface tension gradients, caused by the oil film viscoelastic reaction to the sea surface deformations. When Marangoni and gravity-capillary waves share the same wavenumber for a given frequency, a maximum in the damping occurs. This theory allows for the evaluation of a dampening factor typically affecting waves with wavelength lower than 1 meter. The value of the dampening factor is related to the chemical-physical characteristics of the specific oil pollutant. However, due to non-linear wave interactions, also waves at different wavelength with respect to the Marangoni resonant one are affected by the presence of oil. The overall effect is a wind-dependent redistribution of energy in the directional spectrum in the neighborhood of the dampening frequency [7]. These involved wind-dependent resonant mechanisms differentiate the dampening behavior determined by the presence of oil films from the one related to the so-called look-alikes, e.g. wind-falls [7].

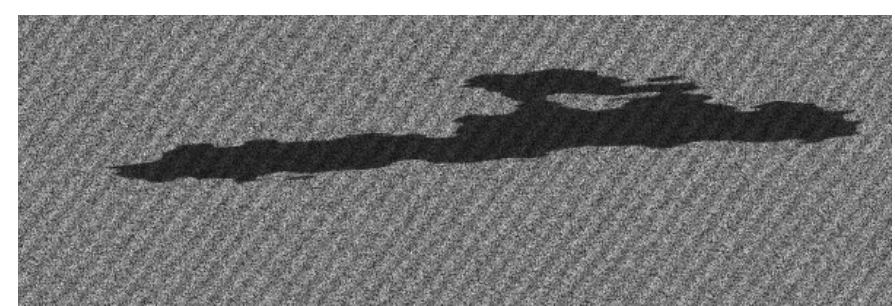

Fig. 1. Simulated SAR image of an oil slick with fractal contour.

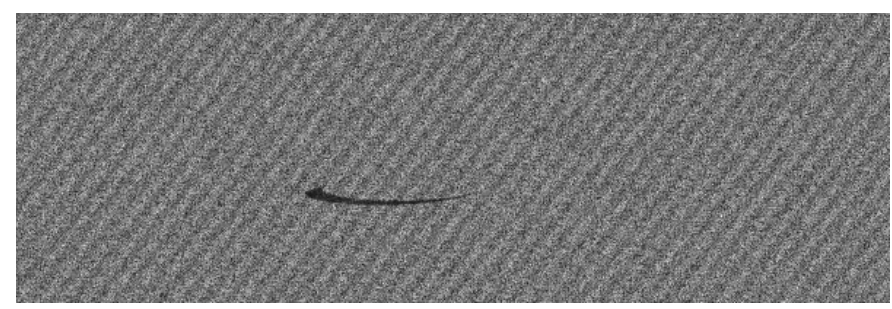

Fig. 2. Simulated SAR image of an oil slick with non-fractal contour.

The Marangoni theory allows for the characterization and simulation of SAR images in presence of oil slicks. As an example in Fig. 1 a simulated SAR image of an oil slick is reported. The slick in Fig. 1 presents a natural shape of the contour. Indeed, its borders are simulated using appropriate fractal techniques [12]. In actual situations the shape of the spill will depend on whether the oil was released from a stationary object or from a moving ship, the amount of oil involved and the wind and current history between the release and the image acquisition. Therefore, the fractal shape can be related either to a naturally occurring spill or to a humaninduced (illegal tank washing) one, after a certain time has passed from the event.

The contour of the slick can also be simulated using rules pertaining to "classical" geometry, as shown in Fig. 2. This shape of the slick is a good approximation for the slicks generated by illegal tank washing soon after the event occurrence. Therefore, analysis of the shape of the slicks via fractal techniques can lead to the identification of illegal actions [12]-[13].

\section{B. Coastal Applications and Ship Detection}

Use of a finite pulse repetition frequency (PRF), and the fact that azimuth antenna pattern (AAP) cannot be designed to be exactly limited to the specified azimuth illumination beamwidth, give rise to the SAR azimuth ambiguity problem. It is responsible for the appearance on SAR images of replicas (or "ghosts") of brilliant points or areas, shifted (mainly) in the azimuth coordinate. These replicas are strongly attenuated, due to the azimuth antenna beam pattern and because they are not well focused. However, when the ghosts are placed on the sea surface, they emerge with respect to the dark background. Accordingly, for coastal or ship-detection applications, ghosts of strong scatterers placed on dry land (e.g., buildings) or also on the sea (e.g., other ships) may cause relevant problems [14][17]. In particular, in ship-detection applications, where automatic Constant False Alarm Rate (CFAR) techniques are frequently used, ambiguity may cause the appearance of false alarms, i.e. the ghosts can be incorrectly identified as actual 
targets [15]. Appropriate countermeasures and pre-processing strategies are thus advisable in presence of significant azimuth ambiguity, in order to avoid significant degradation of the automatic ship-detection system performance [14].

In coastal zones over the sea, and in ocean scenes in very high-resolution images, the ambiguities are mainly due to distributed targets. For these situations, and restricting to the use of single polarization SAR data, the use of techniques based on the concept of selective filtering has been advocated for the mitigation of the azimuth ambiguity phenomenon [16][17]. These techniques use a band-pass filter to select from the whole azimuth spectrum those areas that are less affected by ambiguities, so that this approach can be applied to ghosts due to both point targets and distributed targets, at the expense of a certain resolution loss.

Recently, we developed a method for the filtering of azimuth ambiguities on stripmap SAR images, named "asymmetric mapping and selective filtering" (AM\&SF) method [17]. This method is based on a two-step procedure. In the first step, two asymmetric filters are used to suppress ambiguities due to each sidelobe of the antenna pattern, and the ratios between the original and filtered images are used to produce two binary maps of the ambiguity-affected areas (one for each sidelobe). The transfer function of the filters is obtained using the theory of Wiener filtering [16]-[17]. In the second step, the maps of the ambiguity-affected areas are used to produce a final image in which only the areas affected by the ambiguities are replaced by their filtered versions, obtained via the proper of the two filters cited above. Note that the framework proposed in [17] is able to deal also with the case of high resolution sensors like TerraSAR-X and Cosmo/SkyMed for which, due to the specific combination of azimuth antenna pattern and PRF, the filter discussed in [16] frequently fails.

A relevant example of the filtering results is reported in Fig. 3. The approach of [17] has been proposed as a preprocessing step in the context of ship-detection applications. In particular, in [18] a large set of Cosmo/SkyMed images preprocessed via the proposed azimuth ambiguity filter was provided as input to a ship-detection algorithm. The application of the pre-processing filter was responsible for a drastic decrease of the false alarm rate [18].

\section{CONCLUSION}

In this paper an overview of potential applications of SAR in the ocean monitoring context were presented. In particular, the focus was on single-polarization single-acquisition stripmap images. It has been shown how the analysis and interpretation of SAR images relevant to the ocean surface require the enforcement of appropriate physical models able to describe the sea surface and its interaction with the impinging microwave SAR signal. Based on the introduced models, two main (not standard) applications have been detailed, namely the simulation and identification of oil films on the sea surface and the mitigation of azimuth ambiguity in support of coastal applications and ship detection.

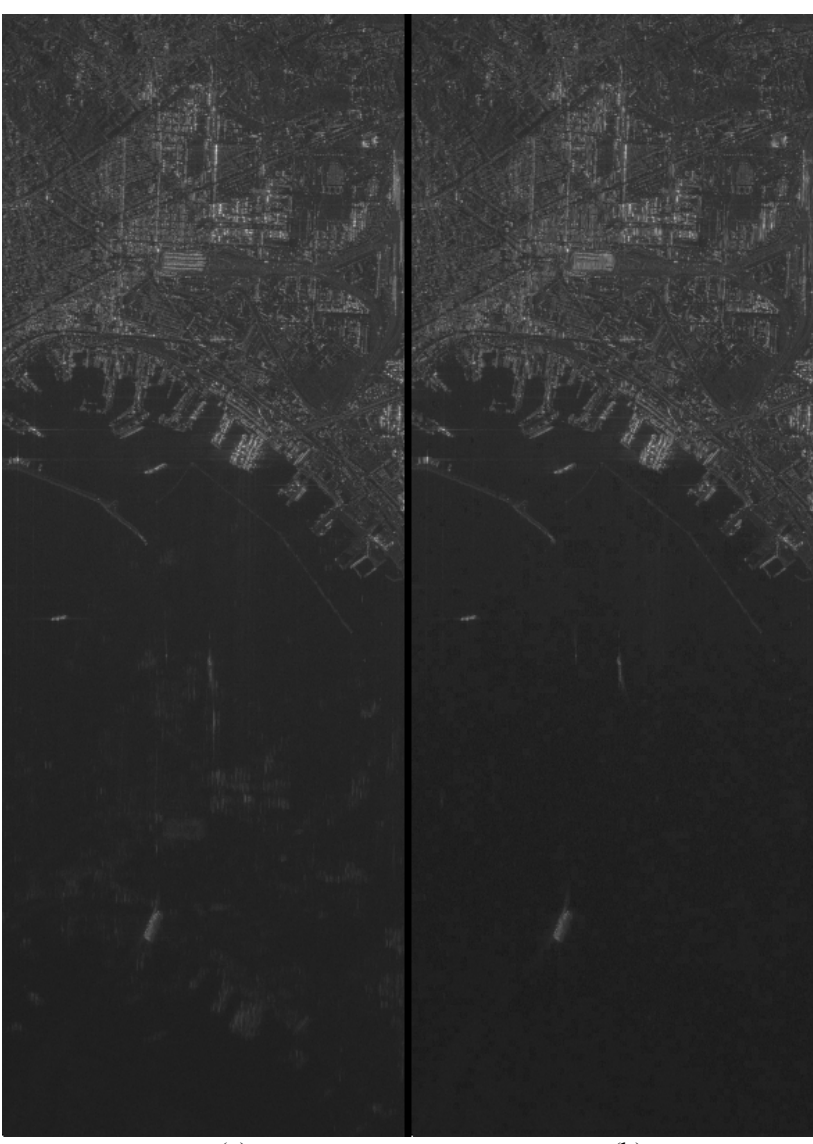

(a)

(b)

Fig. 3. Original Cosmo/SkyMed image of the gulf of Naples (a) and its filtered version (b) (a spatial multilook of 6x6 pixels is applied). Ambiguities due to buildings and man-made areas are visible all over the sea surface in (a), while they are strongly attenuated in (b), where only actual ships are visible on the sea.

\section{REFERENCES}

[1] A. Moreira et al., "A tutorial on synthetic aperture radar," IEEE Geosci. Remote Sens. Mag., vol. 1, no. 1, pp. 6-43, Mar. 2013.

[2] K. Tomiyasu, "Tutorial review of synthetic aperture radar (SAR) with applications to imaging of the ocean surface," Proc. IEEE, vol. 66, no. 5, pp. 563-583, 1978.

[3] G. Di Martino, D. Riccio, and I. Zinno, "SAR imaging of fractal surfaces," IEEE Trans. Geosci. Remote Sens., vol. 50, no. 2, pp. 630 644, Feb. 2012.

[4] G. Franceschetti and D. Riccio, Scattering, Natural Surfaces and Fractals. Burlington, MA: Academic Press, 2007.

[5] G. Franceschetti, M. Migliaccio, and D. Riccio, "On ocean SAR raw signal simulation," IEEE Trans. Geosci. Remote Sens., vol. 36, no. 1, pp. 84-100, 1998.

[6] G. Di Martino, A. Iodice, D. Riccio, and G. Ruello, "Equivalent number of scatterers for SAR speckle modeling," IEEE Trans. Geosci. Remote Sens., vol. 52, no. 5, pp. 2555-2564, 2014.

[7] G. Franceschetti, A. Iodice, D. Riccio, G. Ruello, and R. Siviero, "SAR raw signal simulation of oil slicks in ocean environments," IEEE Trans. Geosci. Remote Sens., vol. 40, no. 9, pp. 1935-1949, 2002.

[8] G. Neumann and W.J. Pierson, Principles of Physical Oceanography. Englewood Cliffs, NJ: Prentice-Hall, 1996.

[9] E. Jakeman and P. N. Pusey, "A model for non-Rayleigh sea echo," IEEE Trans. Antennas Propag., vol. AP-24, no. 6, pp. 806-814, 1976. 
[10] Merv Fingas and Carl Brown, "Review of oil spill remote sensing," Marine pollution bulletin, vol. 83, no. 1, pp. 9-23, 2014.

[11] W. Alpers and H. Huhnerfuss, "The damping of ocean waves by surface films: A new look at an old problem," J. Geophys. Res., vol. 94, pp. 6251-6265, 1989.

[12] A. Danisi, G. Di Martino, A. Iodice, D. Riccio, G. Ruello, M. Tello, J. J. Mallorquí, and C. Lopez-Martinez, "SAR simulation of ocean scenes covered by oil slicks with arbitrary shapes," in Proceedings of IGARSS 2007, pp. 1314-1317.

[13] R. Coscione, G. Di Martino, A. Iodice, D. Riccio, and G. Ruello, "Multifractal analysis of oil slicks on SAR images," in Proceedings of SPIE 2011, vol. 8179, pp. 81790H7/1-81790H/8.

[14] S. Brusch, S. Lehner, T. Fritz, M. Soccorsi, A. Soloviev, and B. van Schie, "Ship surveillance with TerraSAR-X," IEEE Trans. Geosci. Remote Sens., vol. 49, no. 3, pp. 1092-1103, Mar. 2011.

[15] D. J. Crisp, "The state-of-the-art in ship detection in synthetic aperture radar imagery," Intell., Surveillance and Reconnaissance Div., Inf. Sci. Lab., Def., Sci. Technol. Org., Edinburgh, S.A., Australia, May 2004, Res. Rep. DSTO-RR-0272.

[16] A. Monti Guarnieri, "Adaptive removal of azimuth ambiguities in SAR images," IEEE Trans. Geosci. Remote Sens., vol. 43, no. 3, pp. 625-633, Mar. 2005.

[17] G. Di Martino, A. Iodice, D. Riccio, and G. Ruello, "Filtering of azimuth ambiguity in stripmap synthetic aperture radar images," IEEE $J$. Sel. Top. Appl. Earth Obs. Remote Sens., vol. 7, no. 9, pp. 3967-3978, 2014.

[18] C. Avolio, M. Costantini, G. Di Martino, A. Iodice, F. Macina, G. Ruello, D. Riccio, and M. Zavagli, "A method for the reduction of shipdetection false alarms due to SAR azimuth ambiguity," in Proceedings IGARSS '14, 2014, pp. 3694-3697. 